\title{
EDITORIAL
}

\section{Debating the definition of airflow obstruction: time to move on?}

\author{
M.R. Miller*, O.F. Pedersen ${ }^{\#}$, R. Pellegrino ${ }^{\top}$ and V. Brusasco ${ }^{+}$
}

$\mathbf{T}$ he clinical practice of medicine involves accurate history taking and appropriate clinical examination, followed by the formulation of possible diagnoses to account for the patient's condition. This usually leads to investigations being undertaken to confirm or refute the possible diagnoses. These investigations may be of a visual nature, such as imaging (radiology, magnetic resonance imaging or ultrasound) or endoscopy, or may be the measurement of physiological indices, such as blood pressure, height, weight, lung function or measurements from blood tests. For most measurements, clinicians are used to the concept of accepted normal ranges, which may be different for males and females. These ranges may be quite distinct and relatively invariable. For other measurements the situation is less clear, e.g. blood pressure, for which the acceptable ranges may be defined by their relationship to survival and risk of adverse outcome [1].

In lung function tests, the normal ranges have usually been obtained from healthy nonsmoking subjects and are dependent on a number of other aspects related to the individual subject in question, namely their age, sex, height and ethnic background [2]. This complicates the way in which a clinician can quickly interpret whether a subject's result is abnormal. The American Thoracic Society/European Respiratory Society task force on the standardisation of lung function testing has recommended that a given test is said to be abnormal when the measured value is below the lower limit of normality (LLN), defined as 1.645 standard deviations below predicted [3]. However, an alternative way to define a lower limit for deciding on the presence of airflow obstruction for chronic obstructive pulmonary disease (COPD), based on the ratio of forced expiratory volume in $1 \mathrm{~s}$ (FEV1) to forced vital capacity (FVC) was proposed by the Global Initiative for Chronic Obstructive Lung Disease (GOLD) committee [4], which suggested a fixed cut-off of 0.70 .

In this issue of the European Respiratory Journal, two papers present further evidence that the use of fixed FEV1/FVC cutoffs to define airflow obstruction is unsatisfactory. First, VOLLMER et al. [5] have used the Burden of Obstructive Lung Disease (BOLD) dataset to explore what the effect of using

\footnotetext{
*Dept of Medicine, University Hospital Birmingham NHS Trust, Birmingham, UK. " Institute of Public Health, University of Aarhus, Aarhus, Denmark. AAllergologia and Fisiopatologia Respiratoria, Azienda Ospedaliera S. Cuneo, Cuneo, Italy. ${ }^{+}$Dipartimento di Medicina Interna, Università di Genova, Genova, Italy.
}

CORRESPONDENCE: M.R. Miller, Dept of Medicine, University Hospital Birmingham NHS Trust, Selly Oak Hospital, Raddlebarn Rd, Birmingham B29 6JD, UK. E-mail: martin.miller@uhb.nhs.uk different spirometry criteria for defining COPD was on its observed prevalence. VolLMER et al. [5] confirm that a fixed cutoff of 0.70 significantly overestimates COPD prevalence compared with other methods and, more importantly, they show that other definitions using LLN have less bias related to age, sex and the location where subjects were studied. Using a value below the LLN for both FEV1/FVC and FEV1 to diagnose an obstructive abnormality had the least bias and also the least difference in prevalence between results from pre- and postbronchodilator results.

Secondly, CERVERI et al. [6] report the results of a multicentre study on the spirometric diagnosis of asthma, which compared various fixed cut-off values of FEV1/FVC, ranging between 0.70 and 0.80 , with a statistically defined LLN, in terms of their ability to detect airflow obstruction. The authors used data from the healthy part of the population they surveyed to produce reference equations from which the LLN was defined. This overcame any argument that the choice of reference equation might adversely influence their findings. There were 815 asthmatic subjects aged $20-44$ yrs in their population. Within this age range, LLN for FEV1/FVC was $<0.80$ and $>0.70$ for all subjects. Absence of airflow obstruction was defined as FEV1/FVC being greater than the LLN [3]; thus, all subjects in their dataset with a value $>0.80$ will be nonobstructed and all subjects with a value $<0.70$ will be obstructed.

From their reference equations it can be calculated that the predicted FEV1/FVC is 0.70 at the age of $\sim 52$ yrs. This means that subjects aged $<52$ yrs are more at risk of being misclassified as healthy if $0.7<\mathrm{FEV} 1 / \mathrm{FVC}<\mathrm{LLN}$. These subjects can be regarded as false-negatives for the diagnosis of asthma. Conversely, people aged $>52$ yrs are more at risk of being misclassified as having airflow obstruction, even though they are above the LLN, and are, therefore, false-positives. As asthma is a disease of predominantly young people, the 0.70 criterion may, therefore, fail to diagnose many asthmatics as having obstruction, whereas in COPD, which is a disease of predominantly older people, the opposite is true; namely, too many may get the diagnosis of airflow obstruction without having it. FEV1/FVC declines with age [7], mainly due to an age-dependent loss of elastic fibres in the lungs, which reduces the elastic recoil of lung tissue. This is not per se a pathological process and so any consequent drop in FEV1/FVC cannot simply be ascribed to a disease such as COPD.

Some have claimed that that the virtue of the fixed ratio is that it is easy to remember and to apply, and that this utility 
outweighs the disadvantages of using a statistically defined LLN, which will be dependent on the choice of reference equation [8]. CERVERI et al. [6] quantified the degree of misclassification (false-negatives) in the age group $<52$ yrs and found that using the 0.70 ratio to diagnose airway obstruction found only $57 \%$ of the males and $77 \%$ of the females with values below the LLN. A similar proportion was identified in the Third National Health and Nutrition Examination Survey (NHANES-III) database by HANSEN et al. [9], in which $\sim 50 \%$ of young subjects were misclassified as falsenegatives. If the fixed cut-off value was adjusted to get the optimum sensitivity and specificity the prediction did not become perfect. Furthermore, both the adjustment and the LLN will depend on the reference equations chosen and so this solution is not ideal. It is clear from these two papers $[5,6]$ that the fixed cutoff operates prejudicially with respect to age and sex, and these are areas over which protection against discrimination is enshrined in European Union employment law.

The problem of over-diagnosing COPD by using a fixed cut-off value for FEV1/FVC has also been examined by HANSEN et al. [9]. They reported $\sim 20 \%$ misclassification in older age groups. SWANNEY et al. [7] calculated false-positive test results as a percentage of all positive test results for airway obstruction in three large population-based samples of adults, including smokers and subjects with respiratory symptoms. They found that the LLN varied between prediction equations (57 equations available for males and 55 for females) and applying the GOLD fixed criterion of FEV1/FVC $<0.70$ [4] caused false-positive rates of up to $60 \%$ when applied to entire populations.

The diagnosis of asthma requires symptoms [10], whereas the diagnosis of COPD has often been based only on spirometry [4] given the presence of a provoking factor such as smoking or atmospheric pollution. In asthma, bronchodilatation may help establish the diagnosis, but should not be done prior to diagnostic spirometry because that might remove the bronchoconstriction characteristic for asthma. The opposite is true for COPD. The GOLD criteria require bronchodilatation before diagnostic spirometry in order to reduce the chance of asthmatics being classified as having COPD. Post-bronchodilator testing is seldom carried out, but decreases the number of false-positives [11]. Although the LLN is based on a statistical approach, which is to some extent arbitrary, it gives an estimate of the probability of misclassification. Thus, the use of LLN based on scientific grounds seems definitely preferable to the use of a fixed cut-off justified by simplicity only. False classification of COPD using a fixed cut-off for FEV1/FVC has been shown to be significant by several authors and now
CERVERI et al. [6] have confirmed that using this fixed cut-off is also problematic for looking at asthma in younger age groups.

We conclude that the evidence for using LLN to define airflow obstruction is compelling and does not need further experimental clarification. Some may have argued for the fixed cut-off on the basis of simplicity in application so that registration of disease prevalence can be compared across populations, but such prevalences are clearly spurious. Clinicians work with patients face to face. To declare to a patient that they have airflow obstruction based on a fixed cut-off criterion even though it is known that their results are within the range of statistical normality would not reconcile with good clinical practice. We now must move forward to refine and update our prediction equations so that the most reliable LLN can be obtained.

\section{STATEMENT OF INTEREST}

A statement of interest for V. Brusasco can be found at www.erj. ersjournals.com $/ \mathrm{misc} /$ statements.dtl

\section{REFERENCES}

1 Frohlich ED. Reappearance of the J-shaped curve. Hypertension 1999; 34: 1179-1180.

2 Hankinson JL, Odencrantz JR, Fedan KB. Spirometric reference values from a sample of the general US population. Am J Respir Crit Care Med 1999; 159: 179-187.

3 Pellegrino RC, Viegi G, Brusasco V, et al. Interpretive strategies for lung function tests. Eur Respir J 2005; 26: 948-968.

4 Global Initiative for Chronic Obstructive Lung Disease (GOLD). Global Strategy for Diagnosis, Management and Prevention of COPD. GOLD, 2007. Available from: www.goldcopd.org

5 Vollmer WM, Gíslason p, Burney P, et al. Comparison of spirometry criteria for the diagnosis of COPD: results from the BOLD study. Eur Respir J 2009; 34: 588-597.

6 Cerveri I, Corsico AG, Accordini S, et al. What defines airflow obstruction in asthma? Eur Respir J 2009; 34: 568-573.

7 Swanney MP, Ruppel G, Enright PL, et al. Using the lower limit of normal for the FEV1/FVC ratio reduces the misclassification of airway obstruction. Thorax 2008; 63: 1046-1051.

8 Mannino DM. Should we be using statistics to define disease? Thorax 2008; 63: 1031-1032.

9 Hansen JE, Sun XG, Wasserman K. Spirometric criteria for airway obstruction: use percentage of FEV1/FVC ratio below the fifth percentile, not $<70 \%$. Chest 2007; 131: 349-355.

10 Bateman ED, Hurd SS, Barnes PJ, et al. Global strategy for asthma management and prevention: GINA executive summary. Eur Respir J 2008; 31: 143-178.

11 Johannessen A, Omenaas ER, Bakke PS, et al. Implications of reversibility testing on prevalence and risk factors for chronic obstructive pulmonary disease: a community study. Thorax 2005; 60: 842-847. 